\title{
Labyrinthe
}

$27 \mid 2007$ (2)

La fin des disciplines?

\section{Du Seminar au séminaire}

\section{Marc Aymes}

\section{OpenEdition}

Journals

Édition électronique

URL : https://journals.openedition.org/labyrinthe/2011

DOI : 10.4000/labyrinthe.2011

ISSN : 1950-6031

Éditeur

Hermann

\section{Édition imprimée}

Date de publication : 4 juillet 2007

Pagination : $37-58$

ISBN : 978-2-9526131-4-9

\section{Référence électronique}

Marc Aymes, «Du Seminar au séminaire », Labyrinthe [En ligne], 27 | 2007 (2), mis en ligne le 25 mars 2011, consulté le 26 avril 2022. URL : http://journals.openedition.org/labyrinthe/2011 ; DOI : https:// doi.org/10.4000/labyrinthe. 2011

Propriété intellectuelle 


\title{
DU SEMINAR AU SÉMINAIRE
}

\author{
Marc AYMES \\ marc.aymes@voila.fr
}

Que cherchons-nous au juste? Des gestes d'indiscipline. Un faire autant qu'un dire. Soit ici un exemple ${ }^{1}$ : le geste d'une recherche en bande organisée qui a pour nom d'usage séminaire.

\section{DU-AU}

Je dis «Du Seminar au séminaire» : que faut-il entendre par là? Avant même que d'avoir la moindre idée de ce que sont les séminaires en question, le lecteur aura perçu un sens d'historicité dans ce «du... au... ». Ou, au moins, un simulacre d'historicité: tout se passe comme s'il se produisait une évolution, une transformation linéaire, une migration progressive d'une scène, d'une langue à une autre. Et d'un tel processus, l'histoire serait une connaissance à notre portée.

Je ne sais si cette approche historique vaut pour comprendre les mutations de l'espèce seminarium. Sans exclure pareille éventualité, je veux plutôt travailler à en dégager certaines contemporanéités.

1. Contemporanéités: cela signifie que la formule «du... au...» ne doit pas nécessairement se ramener au commode déroulé d'une histoire chronologique, mais que cependant la question posée comporte bel et bien une dimension historique. Ầ savoir, qu'il est toujours possible d'identifier des moments et des lieux clés où, et quand, des séminaires furent investis de formes particulièrement significatives. L'hypothèse est que ces cadres temporels et spatiaux - en un mot, ces contextes - nous mettent en présence de situations privilégiées pour comprendre, à la manière idéaltypique, ce qui se passe dans un Seminar ou dans un séminaire. Je distingue, par ordre chronologique d'entrée en scène, trois de ces contextes:

1. Ou du moins ce que nous pouvons espérer faire passer pour tel; on verra ce qu'il en est. 


\section{Labyrinthe, $n^{\circ} 27$}

En Prusse au XVIII ${ }^{e}$ siècle - et durant ses dernières décennies en particulier. C'est alors qu'a lieu une restructuration majeure des institutions d'enseignement et de recherche. Le Seminar de philologie créé à Göttingen en 1738 met en œuvre deux dynamiques sans précédent: «Il intégrait certains traits des sociétés savantes privées et des séminaires pédagogiques [qui avaient été instaurés auparavant], et il adaptait l'idée du séminaire théologique financé par l'État aux facultés des arts et de philosophie ${ }^{1}$ ». Tel qu'il est instauré en 1787 au sein de l'université de Halle, «un séminaire à ce stade signifiait un institut doté d'un budget de financement - dont l'essentiel était consacré à des bourses pour ses participants ${ }^{2} »$. Cette Institutionalisation de la recherche «contribua à transformer des entités corporatives et collégiales en agences bureaucratiques ${ }^{3}$.»

Dans les États-Unis des années 1860-1890. Le «séminaire de recherche» est la marque de fabrique de l'idée d'université telle qu'elle voit le jour, avec une cohérence sans précédent, sur la Côte Est à cette période. Une particularité de cette conception, encore une fois, est qu'elle «fusionne un style d'enseignement particulier avec un mode spécifique de financement ${ }^{4} »$.

En France dans les années 1950-1970. Cette période est marquée par les mouvements de répression et de lutte intestine qui traversent les diverses «écoles» et «causes» psychanalytiques en place; elle connaît ensuite le bouleversement de la «prise de parole» dont mai 1968 marque l'irruption. Plusieurs figures éminentes de ces événements intellectuels et politiques, tels Jacques Lacan ou Michel de Certeau - lui-même séminariste -, se trouvent à l'époque non seulement étroitement associées, mais directement impliquées dans des initiatives qui relèvent (nous verrons comment) du séminaire.

2. Voici à présent que, coupant court à la simplicité de cette frise en trois temps, le mot contemporanéités oblige aussi à envisager la possibi-

1. William Clark, Academic Charisma and the Origins of the Research University, Chicago, University of Chicago Press, 2006, p. 159: «it integrated aspects of the private societies and pedagogical seminars [that had already existed so far], and it translated the notion of the state-funded theological seminar(y) into the arts and philosophy faculty.» Notons le i grec entre parenthèses, jeu graphique intraduisible en français : nous reviendrons plus loin sur ce lien entre le séminaire des religieux et celui des universitaires.

2. Ibidem, p. 127: «a seminar at this point meant a budgeted institute - the budget mostly went for scholarships for the seminars' participants. »

3. Ibidem, p. 162: «the philology seminars helped transform corporate and collegial academic entities into bureaucratic agencies. »

4. Ibidem, p. 158: «The seminar fused a particular style of teaching with a particular method of funding.» 


\section{$D u \ll$ Seminar » au séminaire}

lité d'une pluralité simultanée de configurations appelées «séminaire». Plusieurs Seminar ou séminaires peuvent avoir été instaurés dans le même temps, développés distinctement, et confrontés avec fruit. La question qui se pose alors est celle de la migration internationale, de la transposition et de la traduction, de l'interpolation et de la réappropriation. Entre autres indices, il y a le trait d'union appuyé que les penseurs de «l'université» aux États-Unis dans les dernières décennies du XIX ${ }^{\mathrm{e}}$ siècle ont tracé entre le Seminar (en allemand) et le seminar (en anglais): la notion du «séminaire de recherche», telle qu'elle fut mise en œuvre dans le contexte académique nord-américain d'alors, renvoyait en effet explicitement à un idéal de «la recherche abstraite, suivant ce que l'on croyait être le modèle allemand à l'état pur ${ }^{1}{ }^{1}$. L'idée donc est de suivre à la trace ces mouvements de transposition.

De sérieux problèmes de langue et de traduction sont à prévoir. Trois langues, au moins, entrent en effet en jeu : l'allemand, l'anglais et le français. Seminar, seminar, séminaire. Lisez ces trois mots à la suite sans trop articuler, vous verrez: c'est le même son ou presque que l'on entend. Et comme en outre ces trois séminaires ne diffèrent que d'une lettre ou deux, voire d'une majuscule uniquement, une banale erreur typographique pourrait suffire à ce que l'on ne sache plus quelle langue je parle. Ce flottement transnational, reconnaissons-le, fait partie du problème. Mais il n'en est que plus important de maintenir chaque occurrence, autant que faire se peut, en version originale: qui a jamais tenté de débrouiller les relations entre des cognats tels que, disons, la conférence et la conference, sait combien il est périlleux de chercher à traduire les idiomes académiques, tant ils comptent de faux amis.

\section{DOUBLE TRANCHANT}

Mais au fait: qu'est-ce qu'un séminaire? Est-il seulement pertinent de poser la question de cette manière? Comme souvent, il semble plus opportun de tester les limites et les bords de la signification prêtée au mot, plutôt que de chercher à en extraire la substance présumée. Aussi les

\footnotetext{
1. Laurence R. Veysey, The Emergence of the American University, Chicago, Chicago University Press, 1965 , p. $12: \ll[\ldots]$ the goal of abstract research on what was believed to be the pure German model.»
} 


\section{Labyrinthe, $n^{\circ} 27$}

possibilités qui viennent à l'esprit lorsque je dis «séminaire» (ou «Seminar», etc.) sont-elles plusieurs. Toutes renvoient à ce qu'il se passe, croit-on savoir, lorsqu'un séminaire a lieu. Pourtant, aucune ne suffit jamais à le définir tout à fait: ce sont des parties prenantes qui cependant demeurent accidentelles. En d'autres termes: voici des quasipropriétés nécessaires qui n'en demeurent pas moins insuffisantes; certaines même sont l'exception plutôt que la règle. Passons donc en revue quelques-unes de ces positions problématiques auxquelles je pense, sans craindre de commencer par les plus élémentaires.

\section{Où il s'avère que les propriétés supposées du séminaire en sont des accidents, à moins que ce ne soit l'inverse}

Position - Un séminaire est un événement oral, ce que l'on pourrait littéralement appeler un acte de parole.

Problème - Ce critère n'est (qui l'eût cru?) en rien suffisant. Le discours d'un dictateur, en effet, fait tout autant fond sur les puissances de l'oralité.

Position - Un séminaire est un événement de conversation (c'est-àdire qu'il implique non pas un, mais plusieurs sujets parlants en interaction), voire ce que la langue anglaise appelle une conversation piece (c'est-à-dire le moment où un fait inhabituel pousse des individus étrangers les uns aux autres à commencer une discussion).

Problème - Ce n'est ni suffisant ni nécessaire. Semblable conversation piece peut aussi bien se produire au cours d'un sit-in - ou bien dans une salle de cinéma lorsque soudain la pellicule quitte le chemin des bobines, et s'enflamme à l'instant précis où le couteau de boucher tranchait le rideau de douche. Et puis il est des séminaires qui non seulement ont lieu sous forme de monologues, mais sont même explicitement conçus comme tels ${ }^{1}$.

\footnotetext{
1. Ce que revendique par exemple Daniel S. Milo, concernant son enseignement à l'École des hautes études en sciences sociales (Paris) : «je n'accepte pas dans mon séminaire que quelqu'un ouvre le bec. Ça fait des années que c'est la règle écrite: chez moi on se tait. Les étudiants le savent. Ils ont le droit de poser des questions parce qu'ils n'ont pas compris. Mais ce n'est pas un dialogue entre nous. Jamais.» (« Pensez, dix minutes », Labyrinthe $\mathrm{n}^{\circ}$ 26, 2007, p. 83)
} 


\section{$D u \ll$ Seminar » au séminaire}

Position - Un séminaire est une entité collective, le lieu où se donnent rendez-vous un nombre plus ou moins important d'individus.

Problème - Encore une fois, c'est également le cas d'un sit-in.

Position - Un séminaire est investi d'un caractère gratuit et original, récalcitrant à l'assignation d'une fonction professionnelle. Autrement dit, il forme plutôt qu'il ne qualifie.

Problème - Un séminaire aussi archétypal (j'y reviendrai) que celui de Jacques Lacan fut longtemps tenu, entre 1953 et 1963, à la clinique SainteAnne, et s'adressait alors clairement à des psychanalystes professionnels; c'est ensuite que Lacan le déclara virtuellement ouvert à «tous » ${ }^{1}$.

Position - Les séminaires ont à voir avec la mise en œuvre d'un certain idéal de recherche. Telle est la conception développée au sein des universités fondées aux États-Unis dans la seconde moitié du XIX siècle: celle d'une recherche qui «offre une instruction avancée, non professionnelle, à des étudiants dotés des qualifications requises, dans divers secteurs des lettres et des sciences ${ }^{2} \gg$. Conséquence:

le seminar devint le principal lieu où l'ethos du Romantisme investit le monde universitaire. On en vint à exiger des étudiants avancés ou graduate qu'ils se livrent à un travail académique original, c'est-à-dire à de la recherche, d'abord dans les seminars, puis dans les laboratoires. C'est en vertu du même processus historique que les undergraduates se virent consignés aux rigueurs du système de notation ${ }^{3}$.

1. Jacques Lacan, Mon Enseignement, Paris, Seuil, coll. «Champ freudien», 2005, p. 79-80: «Je parlais pour des gens que cela intéressait directement, des gens précis qui s'appellent des psychanalystes. Cela concernait leur expérience la plus directe, la plus quotidienne, la plus urgente. C'était expressément fait pour eux, cela n'a jamais été fait pour personne d'autre. Mais il est vrai que je m'étais aperçu que cela pouvait aussi intéresser des gens à qui ça ne s'adresse pas et que cela ne concerne pas du tout. » Voir aussi, du même auteur, Télévision, Paris, Seuil, 1974, p. 9-10.

2. Citation du Register de la Johns Hopkins University (1877-1878), d'après Laurence R. Veysey, op. cit., p. 149 (souligné dans l'original) : «The Johns Hopkins University provides advanced instruction, not professional, to properly qualified students, in various departments of literature and science.» 3. William Clark, op. cit., p. 141-142: «The seminar proved to be the central site in which the Romantic ethos of originality took hold of academics. Original academic work, that is, research, came to be demanded of advanced or graduate students in the seminars, and then also in the labs. This all happened as part of the same historical process that consigned undergraduates to rigors of the grading system. » 
Problème, pourtant - Certains séminaires n'ont rien à voir avec de telles ambitions de recherche pour étudiants avancés. Par exemple le Seminar pédagogique non universitaire créé à Berlin en 1787 (l'année même où était installé le Seminar de l'université de Halle), afin de former «les futurs maîtres des collèges et écoles secondaires de préparation à l'université ${ }^{1}$ ». Tout séminaire, donc, n'est pas nécessairement séminaire de recherche.

Position (une dernière) - Un séminaire requiert, pour sa prospérité, d'avoir lieu en milieu académique. Autrement dit, il repose sur certains modes de production, de transmission et de valorisation du savoir, ayant atteint un haut degré de distinction et d'institutionnalisation. Plusieurs métaphores au long cours soulignent cette spécificité du séminaire comme lieu d'un savoir formé hors de la vie mondaine. Par son étymologie latine, seminarium renvoie à l'image de la pépinière: on la retrouve dans le rapport que rédige le ministre et universitaire prussien Friedrich Gedike en vue de la fondation du Seminar de Berlin en 1787 - rapport intitulé «Zur Einrichtung einer Pepiniere von Schullehrern für gelehrte Schulen ${ }^{2} »$. Plus souvent encore, le séminaire se trouve associé à son homonyme religieux, de quelques siècles son aîné - et le modèle du séminariste de fournir aux concepteurs du séminaire académique tout un ensemble de symboles et d'idées. L'École normale supérieure fut souvent qualifiée, par exemple, de «noviciat universitaire», de «cloître laïque» ou encore de «monastère laïque ${ }^{3} »$. Cause ou effet, l'architecture est en tout cas sans doute pour quelque chose dans cette généalogie méditative. Dessinez quatre ailes refermées en carré sur une cour silencieuse: saurez-vous retrouver les innombrables réminiscences de ce plan-là dans la pierre des universités d'autrefois, comme dans le béton de certains campus plus récents?

Seulement voilà, problème - Certains séminaires n'ont lieu dans les murs d'aucune institution académique. Les premières séances de Lacan, entre 1951 et 1953, se déroulèrent dans sa salle de séjour, au 5, rue de Lille

1. Ibidem, p. 126.

2. D'après Pascale Hummel, Humanités normaliennes. L'enseignement classique et l'érudition philologique dans l'École normale supérieure du XIXe siècle, Paris, Les Belles Lettres, 1995, p. 61.

3. Louis Liard, L'Université de Paris, Paris, H. Laurens, 1909, p. 118; Alfred Mézières, Au temps passé, Paris, Hachette, 1906, p. 46; Édouard Herriot, Jadis, Paris, Flammarion, 1948, p. 64 (citations d'après Pascale Hummel, op. cit., p. 60). 
à Paris. Les enseignements dispensés par Christian Gottlob Heyne (à Göttingen) en archéologie, ou par Emmanuel Kant en anthropologie, tenaient davantage de la leçon privée que des cours magistraux qui s'en inspirèrent ensuite ${ }^{1}$. Et que dire du Privatseminar organisé à Vienne - puis, après son exil aux États-Unis en 1940, transplanté à New York par l'économiste Ludwig von Mises² ? Entre 1920 et 1934, le séminaire se tint chaque année d'octobre à juin, tous les quinze jours, dans son bureau à la Chambre de commerce, entre dix-neuf et vingt-deux heures environ; puis les participants partaient se restaurer dans une trattoria du centre ville, avant que les plus irréductibles (von Mises compris) ne se dirigent, autour de minuit, vers le café Künstler, établissement prisé des économistes viennois à l'époque. La dénomination du Seminar, calquée sur le grade universitaire Privatdozent, l'indique assez: l'enseignement de von Mises ne délivrait aucun titre académique, et n'était officiellement accrédité ni par l'Université ni par la Chambre de commerce de Vienne ${ }^{3}$. C'est dire que des séminaires ont prospéré dans la plus complète intimité, «en dehors de toute publicitét ${ }^{4}-$, bref, loin de toute institution académique.

\section{Pas mon type}

Nonobstant les questions de plurilinguisme et de traduction relevées plus haut, il apparaît donc que nombreux sont les séminaires hébergés sous ce nom. L'aveu s'impose: je ne sais pas ce qu'il en est pour vous, mais en ce qui me concerne j'ai souvent du mal à savoir ce que séminaire

\footnotetext{
1. William Clark, op. cit., p. 153.

2. Voir Ludwig von Mises, The Historical Setting of the Austrian School of Economics, New Rochelle, Arlington, 1969, p. 4-5; et Earlene Craver, Axel Leijonhufvud, «Economics in America: the continental influence», History of Political Economy, 19/2 (été 1987), p. 173-182, ici p. 175.

3. Ludwig von Mises, Erinnerungen, Stuttgart/New York, Gustav Fischer Verlag, 1978, p. 64 ; Murray N. Rothbard, Ludwig Von Mises : scholar, creator, hero, Auburn, Ludwig Von Mises Institute, 1988, p. 13-18.

4. Comme le déclarait Martin Heidegger chez son hôte provençal René Char en 1969. Voir Questions $I V$, Paris, Gallimard, 1976, p. 286-287 (l'édition française est la version originale des comptes rendus de ces séminaires provençaux tenus au Thor en 1966, 1967 et 1969) : «Il s'agit, à quelques-uns, inlassablement, de travailler en dehors de toute publicité à maintenir vive une pensée attentive à l'être, tout en sachant que ce travail doit viser à fonder, dans un lointain avenir, une possibilité de tradition - étant bien entendu que ce n'est pas en dix ou vingt ans qu'on peut mettre de côté un héritage bimillénaire.»
} 
signifie $^{1}$. Aussi me semble-t-il particulièrement ardu de mettre au jour, par-delà l'hétérogénéité du mot, un hypothétique sens commun. Mais disant cela, suis-je en train de prendre le séminaire en défaut? Ou bien l'impropriété du séminaire, ses accidents permanents, ne seraient-ils pas la marque de la part d'indiscipline qui s'y joue?

Voyons cela, en explorant une autre manière de faire face à la difficulté de tirer au clair les propriétés du séminaire: la recherche d'une typologie. C'est la voie adoptée par Henri Meschonnic:

Évoquer le travail du séminaire conduirait à une typologie des séminaires. [...] Deux types radicalement contraires me paraissent orienter les variations individuelles. J'appellerais talmudique le type de fonctionnement qui est un échange de la parole sans autre hiérarchie que celle de la maîtrise, où circule seule l'argumentation. Le discours y est celui d'une pluralité de sujets, une confrontation réciproque et non la formation de disciples. Un exercice de la démocratie: c'est le discours, tous deux liés et inachevables. Et il y a le séminaire d'autorité, celui du maître d'autorité. Il est grec-allemand comme la philosophie. Il ne tient que de fondre ensemble l'autorité et la maîtrise. Il est charismatique. C'est le séminaire de Heidegger par exemple. Et les séminaires des maîtres de vérité parisiens².

Quels que soient ici les «maîtres de vérité parisiens» raillés par Meschonnic, et les mérites de la ligne de partage qu'il trace, l'important est de souligner les connotations axiologiques de sa typologie. L'avantage est clairement donné, en effet, au type de séminaire qui ne vise à former aucun disciple - à un séminaire indiscipliné donc, dans un autre sens du mot encore. Cette indiscipline-là suppose de marquer la différence entre la «maîtrise» et l' «autorité», non sans renvoyer aussi à un contraste entre l'expérience démocratique du texte et le culte d'une personnalité détentrice de vérité. D'un côté, le scrupule presque ritualiste du commentaire mot à mot, plongeant dans les entrailles de textes eux-mêmes faits

\footnotetext{
1. Manière de paraphraser la déclaration liminaire de Laurent Dubreuil lors du colloque qui a formé la matrice du présent numéro: «I don't know for you, but I often don't know what interdisciplinarity means» (texte anglais prochainement publié en ligne : se reporter à http://www.einaudi.cornell.edu/french_studies/conference/undisciplined_knowledge).

2. Henri Meschonnic, «Enseignement, séminaire», Esprit, 11-12 (novembre-décembre 1978), p. 182 187, ici p. 185 (souligné dans l'original).
} 
d'autres textes (à l'exemple du Talmud) - pratique qui, nonobstant l'identification par Meschonnic de la tradition allemande à la "philosophie», remonte très ouvertement aux principes philologiques des Seminar prussiens. De l'autre côté, il y a le séminaire en version autorisée, originale mais sous-titrée, qui à l'impersonnalité de la critique textuelle substitue les forces d'un savoir charismatique ${ }^{1}$. Disons-le autrement, en saisissant au bond cette volée wébérienne: par contraste avec le séminaire «au charisme», il y aurait un séminaire «bureaucratie compatible ${ }^{2} »$.

Force est cependant de constater que tous les séminaires ne satisfont pas aux critères meschonniciens. C'est le cas par exemple de celui de Michel de Certeau, tenu au département d'ethnologie-anthropologie de l'université Paris-VII dans les années 1970:

je cherche à le «tenir » (comme on «tient» une direction) entre deux manières de donner à un Séminaire une identité répétitive qui exclut l'expérience du temps: l'une, didactique, suppose que le lieu est constitué par un discours professoral ou par le prestige d'un maître, c'est-à-dire par la force d'un texte ou par l'autorité d'une voix; l'autre, festive et quasi extatique, prétend produire le lieu par le pur échange des sentiments et des convictions, et finalement par la quête d'une transparence de l'expression commune. Toutes deux suppriment les différences au travail dans un collectif, - la première en les écrasant sous la loi d'un père, la seconde en les effaçant fictivement dans le lyrisme indéfini d'une communion quasi maternelle. Ce sont deux types d'unité imposée, l'un trop « froid» (il exclut la parole des participants), l'autre trop «chaud» (il exclut les différences de places, d'histoires et de méthodes qui résistent à la ferveur de la communication) ${ }^{3}$.

\footnotetext{
1. Voir à ce sujet Laurence R. Veysey, op. cit., p. 156 (souligné dans l'original, je traduis) : «En vertu de ce que présupposait le raisonnement scientifique par induction, la découverte de la vérité tenait précisément à son caractère impersonnel: deux hommes, quels qu'ils soient, étudiant le même document ou observant la même expérience de laboratoire, étaient supposés parvenir aux mêmes conclusions, et ce consensus fondait la croyance. Le processus ne laissait aucune prise à la personnalité. Mais en pratique, c'est parce qu'ils offraient une proximité des contacts humains entre des étudiants avancés et un individu réputé dans son domaine de recherche, que le séminaire et le laboratoire furent prisés. Si les canons scientifiques mirent un terme, dans une large mesure, à l'idéal du paternalisme moral, le paternalisme intellectuel prit le relais. [...] Dans le contexte américain le succès d'un séminaire universitaire tint, bien souvent, à son caractère charismatique; ceux qui substituèrent du rituel à une approche imprégnée d'émotion furent les moins fructueux. »

2. Dans la mesure où, on l'a vu, les Seminar fondés en terre germanique s'inscrivaient dans «le processus bureaucratique de la départementalisation des facultés des arts et sciences» (William Clark, $o p$. cit., p. 142: «the bureaucratic process of the departmentalization of the arts and sciences faculty»). 3. Michel de Certeau, «Qu'est-ce qu'un Séminaire?», Esprit, 11-12 (novembre-décembre 1978), p. 176-181, ici p. 176-177.
} 


\section{Labyrinthe, $n^{\circ} 27$}

C'est donc que la typologie ne suffit pas: braconnier devant l'Éternel, de Certeau cherche un chemin buissonnier. Or l'enjeu est considérable: si le séminaire doit ainsi être toujours différent à lui-même, quel discours pouvons-nous tenir et soutenir à son propos? Ne suis-je pas voué, tandis que je parle du séminaire, à abandonner ma parole à cette modalité de discours instable qu'est le séminaire en acte - non, pas un discours, plutôt une intervention? Au bout du compte, il semble que le seul moyen de parler $d u$ séminaire soit de s'engager aussitôt dans, ou comme dans, un séminaire. Pour vraiment parler du séminaire, il faudrait tenir séminaire sur le séminaire lui-même.

\section{S MAJUSCULE: LE SÉMINAIRE EN THÉORIE}

Est-ce à dire que les séminaires, ou les seminars, ne puissent être professés ex cathedra? Qu'ils ne donnent pas lieu à discours, à cours magistraux, et à théories?

Quelque chose m'intrigue: le fait que certaines occurrences du mot se voient octroyer un $\mathrm{S}$ majuscule. Encore une fois, le problème tient d'abord à des jeux de langue: en allemand, la majuscule initiale est obligatoire pour tout substantif; en anglais, l'usage l'impose dans les titres d'ouvrages ou d'articles; seul le français lui alloue une valeur idiosyncrasique. Tenons-nous en donc à cette langue-ci.

1. L'apposition d'une majuscule, en français, manifeste le passage du nom commun au nom propre. Or, dans le cas des séminaires dont je vais parler, cette appropriation va de pair avec une envie de théorie. De fait, il existe plusieurs textes qui, donnant dans le genre du vade-mecum, visent à s'interroger sur - et, en un sens, à enseigner - ce qu'est un Séminaire: il y a ainsi le court article de Michel de Certeau "Qu'est-ce qu'un Séminaire?», cité plus haut; dans d'autres proportions, les quelques vingt volumes du Séminaire-monde publié au nom de Jacques Lacan par Jacques-Alain Miller participent de la même ambition ${ }^{1}$. En consentant

\footnotetext{
1. On pourrait être tenté de citer encore le «Au séminaire» de Roland Barthes (initialement paru dans la revue L'Arc en 1974 ; réédité dans Le Bruissement de la langue. Essais critiques IV, Paris, Seuil, 1984, p. 393-404; et dernièrement dans Inculte, ${ }^{\circ}$ 12, février 2007). Mais son propos se distingue largement de ce que j'appelle les théories du Séminaire: outre qu'il évite la majuscule, Barthes souligne que le «au» du titre ne signifie pas seulement la localisation de propriétés à enseigner, mais encore et surtout l'adresse d'un éloge, d'une dédicace au séminaire (ibidem, p. 404).
} 
(ou même en aspirant) à ce que ses propositions soient couchées sur papier, le braconnier s'est fait gardien de chasse, le praticien théoricien. Comment interpréter semblable mouvement, sinon comme une tentative de tourner le singulier ici-et-maintenant du séminaire, afin de le convertir en matériau reproductible pour une lecture sans temps ni lieu donnés, voire pour les cours magistraux (lecture, en anglais) de disciples-devenus-maîtres, sur le Séminaire? Le processus suppose que les lecteurs s'acquittent d'un scrupuleux serment d'allégeance, jurant fidélité à l'autorité qu'ils s'engagent à citer ou à transcrire. L'enjeu en outre est ici d'approprier le séminaire, de le rendre propre, de lui donner un nom pour la postérité. Une nouvelle fois, donc, s'opposent des logiques de savoir impersonnel et de connaissance charismatique.

Dans le cas du Séminaire de Lacan, l'autorité est même double, puisque Jacques-Alain Miller a été reconnu comme co-auteur des ouvrages livrant la transcription autorisée de la parole tenue. Et que dit-il? Certes, il souligne à l'envi que «les méandres de cet enseignement [de Lacan] sont cet enseignement même. L'idée de dégager cet enseignement de ses méandres pour le résumer, ou pour rendre son début contemporain de sa fin, méconnaît profondément son objet». Retenons cette idée de l'impossible contemporanéité du Séminaire à lui-même. Cependant, Miller affirme aussi que la fin du Séminaire lacanien était indiscutablement l'Université: «il faudrait être aveugle pour ne pas s'apercevoir qu'être accueilli par l'Université avait pour [Lacan] une valeur essentielle». Et d'ajouter que «la plus haute fonction de l'Université est bien dans la conservation et l'entretien de ce qui est dit par ce qu'elle appréhende comme l'auteur ${ }^{1} \gg$. La preuve est faite, donc: il y a du mausolée dans ce Séminaire-là. Et pour rester dans le domaine lacanien, cette volonté de perpétuation signale l'emprise d'un nachträglich - d'un «après-coup qui fonde la vérité de ce qui a précédé», comme le disait Lacan lors d'une conférence donnée à la clinique psychiatrique du Vinatier, près de Lyon, en $1967^{2}$. Comment pourrait-il être encore question, une fois cet après-coup passé, d'une ouverture du Séminaire à des interventions supplémentaires et à des interprétations interminables? Un séminaire, en somme, peut tout à fait devenir disciplinaire, imposer une discipline à ses destinataires passés, présents et futurs.

1. Jacques-Alain Miller, Entretien sur le Séminaire avec François Ansermet, Paris, Navarin, 1985, p. 39 et 48 .

2. Jacques Lacan, Mon Enseignement, op. cit., p. 62. 
2. Voilà qui n'épuise nullement les voies ouvertes par la recherche d'une théorie du séminaire. Ouvrons-en une autre: l'idée que celui-ci soit le lieu d'une marge propice à la critique. L'ouverture d'un «espace critique», c'est ainsi que de Certeau définit son expérience à Paris-VII ${ }^{1}$. La formule dessine un lien possible entre le séminaire et l'expérience de la «théorie critique»: elle signifie en effet que «la pratique du groupe doit être déterminée par l'élaboration de ses rapports avec son "extériorité", ou plutôt par sa situation de n'être qu'une procédure de sorties et de rentrées relatives à des localisations sociales, professionnelles, familiales, etc. $^{2}{ }^{2}$. Si un tel séminaire a l'Université pour horizon, celle-ci n'a plus rien de l'institution autorisante envisagée précédemment: il s'agit bien plutôt d'un «lieu de transit» inapproprié, grouillant de clandestins malpropres $^{3}$. Ce jeu du séminaire avec sa vaste «extériorité» est le signe de sa double capacité à interpréter, et par réaction à transformer, le monde social dont il se mêle. "Autrement dit, un Séminaire peut produire des manières de prendre de la distance par rapport à nos tâches et des possibilités d'y retourner autrement ${ }^{4}$.» C'est bien de théorie qu'il s'agit donc, comme de Certeau le souligne in fine: « $\mathrm{Si}$, comme je le crois, la théorie se loge toujours dans un écart par rapport à l'institution, elle trouvera par cette structure plurielle sa condition de possibilité5.» Et comment ceci n'aurait-il pas à voir avec l'indiscipline, puisque tout le défi lancé par le séminaire à son dehors consiste à « cesser de juxtaposer à l'expérience du travail une définition onirique et atopique de champs "propres" ${ }^{»} \gg$ ?

\section{IMPROVISATIONS MINUSCULES: LE SÉMINAIRE EN FICTION}

En guise - pas de conclusion, non, mais - d'ouverture à la discussion, je veux à présent me laisser aller à imaginer la fiction d'un séminaire, ou d'un seminar, parmi d'autres. La parole est donc à un autre «je» que moi.

Une fois de temps en temps, tout se met en place de façon plus ou moins aléatoire (même si mes collègues et moi-même, nous y voyons

\footnotetext{
1. Michel de Certeau, art. cit., p. 178.

2. Ibidem.

3. Ibidem (souligné dans l'original).

4. Ibidem, p. 177.

5. Ibidem, p. 181.

6. Ibidem, p. 179.
} 
l'effet des phases de la lune) et alors, nous avons l'intime conviction que le résultat obtenu est extraordinaire. Mais cela arrive rarement, et le reste du temps, tous les participants s'ennuient, non seulement à entendre les chorus des autres, mais même les leurs.

Le séminaire est extrêmement égalitariste, sans doute à cause du présupposé (qu'il partage avec beaucoup d'autres formes d'art) qu'on ne sait jamais où la foudre va tomber et quel membre du groupe, contre toute attente, peut se révéler valeureux quoiqu'il n'en ait jamais donné le moindre signe auparavant.

Considérons ce qui arrive quand tous les participants ignorent le passé, les réputations, tout sauf la contribution de chacun à l'effort collectif. La règle en matière d'improvisation collective, c'est de traiter les apports de chaque individu sur un pied d'égalité. Dans ces situations, il est de règle de traiter les apports de chacun comme potentiellement supérieurs à ceux des autres. Chaque fois que quelqu'un fait quelque chose de nettement meilleur, tout le monde renonce à ses propres idées et se met immédiatement à travailler sur cette idée plus féconde. Les gens ne prennent pas mille précautions, ils ne convergent pas progressivement par une sorte d'amalgame d'indications subtiles et de suggestions, afin de respecter un semblant d'égalité.

Permettre aux mauvais de jouer avec les bons pour se plier à l'étiquette de la profession, n'est pas sans réelles conséquences. L'improvisation collective exige que chacun soit très attentif aux autres participants et soit prêt à modifier ce qu'il fait, et à réagir aux subtiles indications qui suggèrent une nouvelle direction peut-être intéressante à suivre. Ici, l'étiquette est plus subtile que je ne l'ai laissé entendre jusqu'alors, car tout le monde est conscient que tous ceux (ou presque) qui participent à l'improvisation sont constamment en train de suggérer ce qu'on pourrait faire ensuite, sous forme de mouvements à peine esquissés, de légères variations qui vont dans une direction au lieu d'une autre direction possible. Au fur et à mesure que les gens s'écoutent les uns les autres, certaines de ces suggestions commencent à converger et d'autres, qui s'accordent moins bien avec la direction qui se dessine, sont abandonnées. Ainsi les participants élaborent une direction commune qui, trait caractéristique - comme s'ils avaient tous lu Émile Durkheim -, semble dépasser la somme des individus, comme si elle était douée d'une vie propre.

Interrompons ici ce récit de fiction. Vous, lecteur, commencez peutêtre à vous douter que quelque chose n'est pas d'aplomb. C'est que, je 
l'ai dit, je ne suis pas l'auteur de ce qui précède: j'ai simplement lu, compilé et légèrement altéré des extraits d'un texte intitulé «L'Étiquette de l'improvisation» du sociologue Howard S. Becker ${ }^{1}$. Et figurez-vous que ce texte ne traite nullement d'un séminaire ou d'un seminar, mais de jazz et de jam sessions.

Cela ne revient-il pas au même?

Du Seminar au séminaire... sous ces noms, de multiples situations et institutions de savoir ont trouvé à s'instaurer et à se transmettre. Au reste, il n'est jamais acquis que ces termes institutionnalisés, «Seminar» et «séminaire», soient eux-mêmes indispensables - ou au contraire parfaitement accidentels - dans ce qui advient ${ }^{2}$. Car qu'advient-il, au juste? Un mélange de charisme et d'ironie, de scrupule et de suspens. Bref, ce qu'il se passe dans un séminaire, nul ne le sait vraiment: l'essentiel arrive à l'insu de ses participants ${ }^{3}$. Un savoir sans le savoir, en quelque sorte. Avec, toujours, comme une musique flottant dans l'air" ${ }^{4}$. "C'est comme si, au lieu que ce soient les musiciens qui produisent la musique, ils étaient produits par elle, comme dans le Zen ${ }^{5}$.»

Du Seminar au séminaire... et au-delà, quelle différence cela fait-il? Au vrai, il y a là matière à discussion. C'est à vous, donc. Commençons.

1. Voir Howard S. Becker, «The etiquette of improvisation», dans Mind, Culture, and Activity, 7/3, 2000, p. 171-176, ici p. 171-72 et 175-76. J'ai repris, à quelques interventions près, la traduction française (par Axel Nesme) «L'Étiquette de l'improvisation", publiée dans Howard S. Becker, Propos sur l'art, Paris, L'Harmattan, coll. «Logiques sociales», 1999, p. 117-129 (ici p. 118-121 et 126). Les deux textes sont également accessibles en ligne: http://home.earthlink.net/ hsbecker/Improv.html (visité le 23 avril 2007).

2. Voir ainsi la boutade lancée par Lacan en ouverture de sa conférence «Donc, vous aurez entendu Lacan» (à la faculté de médecine de Strasbourg) en 1968 : «Le genre "conférence” n'est pas le mien. Ce n'est pas le mien parce que je fais tous les huit jours depuis quinze ans quelque chose qui n'est pas une conférence, qu'on a appelé un séminaire au temps de l'enthousiasme, et c'est un cours, mais c'est un séminaire quand même, ça en a gardé le nom. » (Mon Enseignement, op. cit., p. 115).

3. Michel de Certeau, art. cit., p. 176: «la première tâche, dans un Séminaire, est de respecter ce qui ne s'y dit pas, et plus encore ce qui y passe d'insu, donc de modérer l'envie d'articuler, de pousser, de coordonner soi-même les interventions de chacun» (souligné dans l'original).

4. Ce qui nous rapproche de l'insituable chambre rouge dans la série Twin Peaks de David Lynch, dont l'hôte principal, un petit homme venu d'ailleurs, déclare: «Where we're from, the birds sing a pretty song, and there is always music in the air» (épisode 2; Lynch/Frost Productions, 1990). Je suis à cet égard heureux qu'une autre musique (du blues cette fois, plutôt que du jazz) intervienne également dans la contribution d'Anthony Mangeon à ce numéro; voir p. 74.

5. Becker, «L'Étiquette de l'improvisation», art. cit., p. 121. Lacan suit également cette ligne Zen en ouverture de la première leçon du Séminaire: «Le maître interrompt le silence par n'importe quoi, un sarcasme, un coup de pied. C'est ainsi que procède dans la recherche du sens un maître bouddhiste, selon la technique zen. » (Le Séminaire Livre I. Les Écrits techniques de Freud 1953-1954, Paris, Seuil, 1975, p. 7). 


\section{SUPPLEMENT : LECTURES}

\section{Lintervention de la différence}

Certes notre Séminaire a connu des moments d'euphorie communicante ou de "dynamique de groupe", et aussi des moments où venait la demande que, de ma place particulière, je situe et rassemble dans un discours les interventions des participants. S'il est normal que cela arrive, cela ne doit pas être la norme, car elle compromettrait ce qui, dans un groupe, peut être expérience politique de la parole (des rapports discrets de forces), création d'événements dans le temps (des "naissances" grâce à la relation avec l'autre) et production d'un langage dialogal (une communication relative à des différences maintenues) - trois éléments qui vont de pair.

Ma position serait donc plutôt d'expliciter ma place particulière (au lieu de la camoufler sous un discours supposé capable d'englober les autres), d'en offrir le plus d'effets possibles, théoriques ou pratiques, à la discussion du groupe, et réciproquement de réagir aux intervenants d'une manière interrogative qui les pousse à dire leur différence et à trouver dans les suggestions que je peux faire le moyen de la formuler plus fortement. Les "modèles" théoriques proposés ont pour fonction de découper des limites (la particularité de mes questions) et de rendre possible des écarts (l'expression d'expériences et de questions autres). Par là s'amorce le travail commun qui crée des événements: une série de différenciations permet à chacun de spécifier pas à pas son propre chemin dans la masse des informations échangées. 


\section{LES SEMINAR}

In 1787, the year before the Prussian Supreme School Council set up the Abitur [the exam that would become the university entrance examination], two important seminars were founded in Prussia. [Friedrich] Gedike [Prussian minister and academic] had a hand in both. With Gedike himself as the director, a nonuniversity pedagogical seminar was attached to the Friedrichwerdesches Gymnasium in Berlin at which Gedike served as rector. This seminar had the mission of training or certifying future instructors for college or university preparatory secondary schools, the gymnasia in the modern sense.

In the same year, Gedike negotiated with the philologist and professor at the University of Halle, F[riedrich] A[ugust] Wolf, about the foundation of the first university seminar in Prussia in the arts and philosophy faculty. [...] [A] seminar at this point meant a budgeted institute - the budget mostly went for scholarships for the seminars' participants.

William Clark, op. cit., p. 126-127.

En ce qui concerne plus spécifiquement le séminaire philologique, tel qu'il fut pratiqué aux grandes heures du développement de l'Altertumswissenschaft, à Göttingen avec Heyne, à Halle avec Wolf ou à Leipzig avec Hermann, il fut créé sur le modèle du séminaire théologique et, contrairement aux cours professoraux ex cathedra, il était destiné à former des professeurs et non des savants. On raconte d'ailleurs qu'il n'était pas rare que Heyne ou Hermann découragent leurs étudiants de s'engager dans la recherche. Fondé par F. Gedike, qui rédigea en 1787 le plan "Zur Einrichtung einer Pepiniere von Schullehrern für gelehrte Schulen", le Séminaire pédagogique royal de Berlin avait pour mission d'enseigner l'art d'enseigner plus que les matières et les contenus.

Pascale Hummel, op. cit., p. 61. 


\section{DE PRUSSE}

The first seminar at the University of Berlin existed as early as 1830. Thereafter the German seminarium led a somewhat spasmodic existence. It was usually the product of a magnetic individual professor, and it did not become a routine part of the curriculum. [...] [T] he American seminar proved no more automatic in its success than had the German. A considerable variety of procedures for it developed. Students reported on the progress of their researches; they gave book reports; articles and monographs were discussed; long papers were read; or the entire class might give itself over to a minute study of documents. [...] When the seminar did spectacularly succeed, the credit went to the personality of the particular scholar who led the endeavor. (By all indications, this was true in the scientific laboratory as well.) Such a statement may seem obvious enough in retrospect, but in the context of the researcher's creed at the time, it was deeply ironic. According to the premises of scientific induction, truth was achievable precisely because it was impersonal: any two men looking at the same document or observing the same experiment were expected to agree, and this agreement laid the foundation for belief. No room was left in this process for personality. But in practice the seminar and the laboratory were prized for the close human contact they offered between advanced students and a man of major reputation in the field. If the ideal of moral paternalism was largely rejected by the canons of science, intellectual paternalism often replaced it. [...] The successful American academic seminar was likely to be charismatic in quality; the less fruitful one substituted ritual for deeper emotional experience. 


\section{TRÉSOR DE LA LANGUE FRANÇAISE}

A. RELIG. CATH.

1. a) (Grand) séminaire. Établissement d'enseignement supérieur consacré à la formation des jeunes gens qui se destinent à la prêtrise.

Directeur, supérieur du (grand) séminaire; entrer au séminaire. L'aîné qui étudiait pour être prêtre au grand séminaire de Nancy (BARRĖS, Colline insp., 1913, p. 263):

$P$. anal. Séminaire + adj. donnant une précision sur la relig. Établissement destiné à la formation de pasteurs ou de rabbins. Tous deux [Schelling et Hegel] sont sortis (ainsi que Strauss) du séminaire protestant de Tübingen (MICHELET, Journal, 1842, p. 428). [L'exégèse moderne] a renouvelé et vivifié l'intelligence de beaucoup de pages de l'Écriture, forcé les portes des séminaires rabbiniques d'Occident, comme celles des séminaires catholiques eux-mêmes (WEILL, Judaïsme, 1931, p. 80). b) (Petit) séminaire. Internat d'un enseignement religieux secondaire, fréquenté par de jeunes garçons parmi lesquels se recrutent les futurs élèves du grand séminaire. Au petit séminaire, je n'étais qu'un élève médiocre. Au grand séminaire, allez, j'ai fini par lasser tout le monde (BERNANOS, Soleil Satan, 1926, p. 129). Bon nombre d'établissements secondaires [catholiques] constituent en fait des petits séminaires où les jeunes gens qui se destinent à la prêtrise reçoivent l'enseignement classique (Encyclop. éduc., 1960, p. 86).

2. P. méton.

a) Ensemble du personnel et des élèves du séminaire. Un séminaire, en rangs pressés, qui suivait le quai Debilly, mettait une queue de soutanes, couleur d'ocre, dans la clarté diffuse (ZOLA, Page amour, 1878, p. 908). b) Bâtiments abritant cet établissement. Cour, mur du séminaire. L'ancien séminaire Saint-Magloire, où se trouve aujourd'hui l'Institution nationale des Sourds-Muets (JOUY, Hermite, t. 3, 1813, p. 262). Le petit séminaire [...] présente deux façades, brique et pierre (VERLAINE, CEuvres posth., t. 1, Souv. et fantais., 1896, p. 229).

c) Durée des études du petit ou du grand séminaire. Je ne l'avais jamais, depuis le séminaire, sentie si vivante, si pressante [ma vocation sacerdotale] (BILLY, Introïbo, 1939, p. 192). 


\section{$D u \ll$ Seminar au séminaire}

\section{INFORMATISÉ (http://atilf.atilf.fr/) «art. «Séminaire»}

3. Peu usuel, p. anal. Cage où l'on met les poulets à l'engrais. Synon. épinette $^{2}$, mue $^{1}$. Oiseaux que l'on confinait dans un obscur séminaire, afin que durant quelques jours, ils n'y pussent bouger que pour picorer et s'engraisser ainsi de façon anormale (JAMMES, Mém., 1921, p. 114).

B. - P. ext. Établissement, lieu ou milieu où l'on se prépare à une profession, où l'on s'exerce à un art, à une technique quelconque. Synon. pépinière. Depuis sa fondation, Le National est un séminaire d'intrigants et de renégats (PROUDHON, Qu'est-ce que la propriété ? Paris, Lacroix, 1867 [1841], p. 313, note). Le séminaire français de l'architecture à Rome est donc, à notre avis, le pourvoyeur de ces banalités audacieuses et coûteuses qui remplissent nos cités. Et les quelques artistes distingués qui sortent de cette école [...] forment une classe sans influence (VIOLLETLE-DUC, Archit., 1872, p. 166).

- En partic. En Allemagne et dans quelques pays d'Europe, établissement d'enseignement supérieur rattaché à une université. L'Allemagne, où les études de musicologie sont réglées dans un esprit scientifique, a créé des instituts ou séminaires modèles. II n'y a pas d'université, si petite soitelle, qui n'en possède un. De même, en Autriche et en Suisse, à Utrecht en Hollande et, depuis 1937, à Rome (Civilis. écr., 1939, p. 50-2).

C. P. anal.

1. ENSEIGN. SUP. Groupe d'étudiants effectuant un travail pratique de recherche sous la direction d'un enseignant; ensemble des séances de ce groupe. (Dict. $X X^{e} \mathrm{~s}$.).

2. P. anal. Réunion de spécialistes ou de techniciens pour étudier des questions ou résoudre des difficultés concernant leur spécialité ou la vie de leurs entreprises. Plusieurs fois par semaine, tous les chercheurs d'un même groupe se réunissent en un "séminaire", selon la désignation courante de ces réunions, où l'on peut suivre les recherches de chacun et où se mettent en commun les projets, les idées, qui se préciseront au crible d'une critique amicale (LEPRINCE-RINGUET, Atomes et hommes, 1957, p. 68). [Des centres] où sont organisées, dans des sessions communes, des sortes de "séminaires de patrons et d'ouvriers soucieux d'améliorer le climat social et l'efficacité économique des entreprises " (Univers écon. et soc., 1960, p. 44-11).

Prononc. et Orth.: [semine:R]. Ac. 1694, 1718: seminaire, ensuite sé-. 
Étymol. et Hist. 1. 1551 «principe vital d'un phénomène » (LE ROY, Timée de Platon, 87 v ${ }^{\circ}$ d'apr. FEW t. 11, p. 440a); 2. ca 1586 (E. PASQUIER, Lettres, IV, 24, éd. Amsterdam, 1723, t. 2, p. 114b: on leur [aux Jésuites] a donné plusieurs maisons pour instituer la jeunesse, qu'ils appellent aujourd'huy Seminaires, voulans sous ce mot donner à entendre que ce sont pepinieres de la Religion Catholique); 1681 petit seminaire (Serment de l'archevêque de Bordeaux ds Archives hist. de la Gironde, t. 8, p. 294); 1718 p. ext. (Ac. : tout le seminaire se trouve à ce sermon); 3. 1636 p. anal. (MONET, p. 808b: La France est un vrai

Seminaire de geans de guerre); 4. a) 1845 nom porté en Allemagne par divers établissements laïques d'instruction publique (BESCH.); b) 1893 "groupe de travail d'étudiants dirigé par un professeur» (G. PARIS, Le Haut enseignement hist. et philol. en France, p. 29 ds QUEM. DDL t. 15).

\section{ÉTIQUETTE ET}

Once every several months, a lot of things, varying more or less randomly (although my colleagues and I often went in for theories that involved phases of the moon), would come together right, and the results would be extraordinary, we thought and felt. Usually that didn't happen, and everyone involved was bored, not only listening to the other players' choruses, but even to their own.

[...]

Jazz is aggressively egalitarian, probably on the premise (common in many of the arts) that there is no telling where lightning might strike and what unlikely person might turn out to be really good even though they had never showed it before.

[...]

Allowing the bad to play with the good, in deference to professional etiquette, has real consequences. Collective improvisation - not like Keith Jarrett, where one man plays alone, but like the more typical small jazz group - requires that everyone pay close attention to the other players and be prepared to alter what they are doing in response to tiny cues that suggest a new direction that might be interesting to take. The etiquette here is more subtle than I have so far suggested, because everyone understands that at every moment everyone (or almost everyone) involved in the improvisation is offering suggestions as to what might be done next, in the form of tentative moves, slight variations that go in one way rather than some of the other 


\section{Du «Seminar» au séminaire}

Empr. au lat. seminarium « pépinière » (de là l'empr. fr. ca 1600, O. DE SERRES, VI, 4 ds HUG.), fig. « source, origine » (de là ca 1586, PASQUIER, Lettres, IV, 4, t. 2, p. 80b), spéc. en parlant d'un milieu, d'une classe où se recrutent les aspirants à certaines fonctions ([equites] seminarium senatus), de là, depuis le concile de Trente (1545-63), le sens 2 (Conc. Trid. sess. XXIII, decret. ds BLAISE Latin. Med. Aev.). 4 est empr. par l'intermédiaire de l'all. Seminar, sens $4 a$ et b (Duden. Das grosse Wörterbuch der deutschen Sprache, t. 5, 1980, p. 2378).

Fréq. abs. littér. : 793. Fréq. rel. littér. : XIX s. : a) 1 006, b) 1143 ; $X X^{e}$ s. : a) 1 229, b) 1 161. Bbg. MICHEL (P.-G.). "Séminaristes sociaux » et «monde moderne » au début du s. Foi Lang. 1978, t. 2, pp. 293-296.

\section{IMPROVISATION}

possible ways. As people listen closely to one another, some of those suggestions begin to converge and others, less congruent with the developing direction, fall by the wayside. The players thus develop a collective direction which characteristically - as though the participants had all read Emile Durkheim - feels larger than any of them, as though it had a life of its own. It feels as though, instead of them playing the music, the music, Zen-like, is playing them.

[...]

Consider what happens when all the participants ignore the past, ignore reputations, ignore everything but the contribution people make to the collective effort. The rule in conventional improvisation is to treat everyone's contribution as "equally good". The rule in these situations is to treat everyone's contribution as "potentially better" than all the others. Whenever anyone does something clearly better, everyone else drops their own ideas and immediately joins in working on that better idea. People do not move gingerly, gradually converging on some sort of amalgam of hints and implications, thus respecting the fiction of equality.

To be able to do that, everyone involved must have much the same idea of what the better looks like, a common criterion for knowing it when it appears. That suggests that they will have shared a past in which those criteria and their application have been worked out and applied in many cases, which in turn suggests their long-term 
participation in an organized world in which the kind of activity they are improvising is common, probably a professional or quasi-professional world in which ties of occupation bring people together in joint projects.

[...] In talking this way, I have gone beyond the face-to-face situation in which improvising typically occurs and is appreciated to the larger movements and organizations in which those situations are embedded. In so doing, I want to avoid the error often made in analyses of face-to-face interaction, the error of neglecting what is fixed in the situation under study by the influences of these larger entities. The relations between the dynamics of improvisation - the constant attentiveness and shifting focus of the participants, and the sensitivity to all that of an experienced audience and the limits placed on those dynamics by tensions of the organized distribution of knowledge and the rewards connected to it suggest some of the dimensions still to be explored.

Howard S. Becker, «The etiquette of improvisation», art. cit., p. 171-72, 175-76.

C'est peut-être ce que l'on trouve dans certaines expériences de drogues (dans l'expérience de certaines drogues). Sans fumer soi-même (ne serait-ce que par l'incapacité bronchique d'avaler la fumée), comment être insensible à la bienveillance générale qui imprègne certains locaux étrangers où l'on fume du kif? Les gestes, les paroles (rares), tout le rapport des corps (rapport néanmoins immobile et distant) est distendu, désarmé (rien à voir, donc, avec l'iuresse alcoolique, forme légale de la violence en occident): l'espace semble plutôt produit par une ascèse subtile (on peut y lire parfois une certaine ironie). La réunion de parole devrait, me semble-t-il, chercher ce suspense (peu importe de quoi: c'est une forme qui est désirée) [...]. En somme, dans les limites mêmes de l'espace enseignant, tel qu'il est donné, il s'agirait de travailler à tracer patiemment une forme pure, celle du flottement (qui est la forme même du signifiant); ce flottement ne détruirait rien; il se contenterait de désorienter la Loi: les nécessités de la promotion, les obligations du métier (que rien n'interdit dès lors d'honorer avec scrupule), les impératifs du savoir, le prestige de la méthode, la critique idéologique, tout est là, mais qui flotte.

Roland Barthes «Écrivains, intellectuels, professeurs», dans Le Bruissement de la langue. Essais critiques IV, Paris, Seuil, 1984, p. 367-391, ici p. 391 (première parution dans Tel Quel, 1971). 